\title{
Giant optical activity of sugar in thin soap films
}

Janine Emile ${ }^{a}$, Olivier Emile ${ }^{b, *}$, Aziz Ghoufi ${ }^{a}$, Alain Moréac ${ }^{a}$, Federico Casanova ${ }^{a}$, Minxia Ding $^{\mathrm{a}, \mathrm{c}}$, Patrick Houizot ${ }^{\mathrm{d}}$

anstitut, CNRS UMR 6521, Université de Rennes I, Campus de Beaulieu, 35042 Rennes, France

${ }^{\mathrm{b}}$ Laboratoire de Physique des Lasers, URU 435, Université de Rennes 1, Campus de Beaulieu, 35042 Rennes, France

${ }^{\mathrm{c} I n s t i t u t}$ de Chimie de Rennes, CNRS UMR 6226, Université de Rennes I, Campus de Beaulieu, 35042 Rennes, France

${ }^{\mathrm{d}}$ LARMAUR, ERL 6274, Université de Rennes I, Campus de Beaulieu, 35042 Rennes, France

\begin{abstract}
We report on enhanced experimental optical activity measurements of thin soap films in the presence of sugar. This unusual optical activity is linked to the intramolecular chiral conformation of the glucose molecules at the air/liquid interface. Choosing sodium dodecylsulfate (SDS) as a model surfactant and glucose as model sugar, favorable interactions between the anionic group $-\mathrm{OSO}_{3}{ }^{-}$- and glucose molecules are highlighted. This induces an interfacial anchoring of glucose molecules leading to a perturbing influence of the asymmetric chiral environment.
\end{abstract}

Keywords:

Soap film; optical activity; interfacial structure; chirality

${ }^{*}$ Corresponding author.

E-mail address: olivier.emile@univ-rennes1.fr (O. Emile) 


\section{Introduction}

Soap bubbles and soap films are fascinating systems which have long attracted scientific interest. To prolong their lifetime, it is usually suggested to add glycerin or sugar in order to strengthen the bubble or the film and to slow down evaporation or drainage [1-4]. It is known that the increase of the viscosity damps capillary waves, but the arrangement of the surfactant and glycerin molecules at the air/liquid interface remains a highly discussed issue [5-8]. On the other hand, glucose, like most of the molecules of biological importance is a chiral molecule. As a chiral molecule it exhibits optical activity either in solution or in its natural form. Biot was one of the first to measure optical activity of organic substances in solution [9]. Today, glucose optical activity is used to evaluate the quality and the concentration of syrup in the food industry [10,11]. Besides, several attempts have been made to use it to diagnose diabetic diseases $[12,13]$ or in the pharmaceutics industry [14]. Since analytical chemistry has been recently used to determine the composition of soap films [15] one may wonder whether films with sugar added could be probed by measuring the optical activity. The aim of the letter is thus to investigate the optical rotary power of glucose in thin soap films depending on the glucose concentration or on the film thickness and to get more insights into its conformation at the air/liquid interface.

\section{Experimental methods}

The soap solutions consist of an anionic surfactant, sodium dodecylsulfate (SDS, Sigma Aldrich, $99.5 \%$ grade) diluted in pure water. The concentration, $12 \mathrm{~g} / \mathrm{L}$, is far above the critical micellar concentration $(\mathrm{cmc}=2.3 \mathrm{~g} / \mathrm{L})$ and is the same as the one used in previous experiments related to the structure of films confined in a channel $[6,16]$. Sugar is $\alpha$-DGlucose (Sigma Aldrich, 96\% grade). For each experiment, we prepared and used fresh solutions to avoid the hydrolysis of SDS into dodecanol. The experimental setup to measure optical activity includes a diode-pumped green laser (CrystaLaser, beam size at the center of the film, according to its gaussian shape $w=360 \mu \mathrm{m}, \lambda=532 \mathrm{~nm}$ ) and two Glan-Laser Calcite Polarizers (Thorlabs) placed on either side of the sample, using the Malus law. The first one is used as a polarizer, the one behind the sample is used as an analyser. Without the sample, the two polarizers are orthogonal to each other and the light transmission is minimized. With a sample exhibiting optical activity, we measure the rotation $\alpha$ of the 
analyser that corresponds to the minimum of transmission. Optical activities of sugar soap films were measured according to Biot's law: $\alpha=[\alpha] l c$, where $l$ is the length of the traversed optically active medium (in $\mathrm{dm}$ ), $c$ is the concentration in $\mathrm{g} / \mathrm{L}, \alpha$ is the adimensional rotation angle and $[\alpha]$ is the specific rotation (in $\% \mathrm{~cm}^{3} /(\mathrm{g} \cdot \mathrm{dm})$ ). The detector is a usual photodiode connected to a computer. This rotation of the polarization of the light beam is also called circular birefringence since the optical index for the two circular polarizations is different. In all our experiments, we have verified by changing the incident linear polarization of the laser beam that there is no linear birefringence.

We have first checked that the specific rotation for large cells containing glucose solutions is $+64^{\circ} \pm 3^{\circ}(\mathrm{g} . \mathrm{dm})$ in agreement with the values usually reported in the literature. For thin films, we measured the rotation angle only. Using a Hamilton syringe, a small amount of solution is applied to deposit a single film inside a circular tube. The material (glass, Kapton) and the diameter of the tube allow different film thicknesses that were measured separately by optical interferometry $[6,16]$. We measured the transmitted signals of two low power He-Ne lasers $(\lambda=543.5 \mathrm{~nm}$ and $632.8 \mathrm{~nm})$ which cross the center of the film confined in the tube. Using the expression of intensity transmissivity $[6,16]$ and the two laser wavelengths, one can unambiguously indentify the fringe order and then the film thickness. We have also checked that the refractive index $\mathrm{n}$ has the same value for the film and for the corresponding solution. All these experiments are performed at a controlled temperature $\mathrm{T}=20.0 \pm 0.5^{\circ} \mathrm{C}$.

We have also collected Raman spectra of sugar soap films and of dried drops at room temperature (around $20^{\circ} \mathrm{C}$ ) in the $400-1400 \mathrm{~cm}^{-1}$ wavenumber range using a LabRAMHR800 (HORIBA Jobin Yvon) confocal Raman spectrometer equipped with an air-cooled CCD detector. The $632.818 \mathrm{~nm}$ line of a $15 \mathrm{~mW}$ He-Ne laser was used as the excitation source. It is focused on the sample via a x100 ultra long working-distance Olympus objective (diameter of the focused beam spot around $1 \mu \mathrm{m}$, depth of focus around $2 \mu \mathrm{m}$ ). The scattered light is collected in backscattering configuration and the Rayleigh scattering is removed by means of a dielectric edge filter. The wavenumber resolution is around $\pm 1 \mathrm{~cm}^{-1}$ per pixel. The deposited drops on microscope slide have a thickness of about $5 \mu \mathrm{m}$. Systematic Raman measurements were made on SDS, glucose and SDS+glucose mixtures. 


\section{Optical activity and Raman spectra}

Optical activities of SDS+glucose films versus thickness are shown in figure 1.a for various glucose concentrations. Curiously, whatever the glucose concentration, the optical activity is unexpectedly large for thin films, of the order of a few tenths of a degree. The extrapolation of Biot's law in solution to soap films would have lead to undetectable rotations. Moreover, beyond a thickness of $200 \mathrm{~nm}$, whatever the concentration, it remains constant. We also performed measurements on films made of SDS only at concentrations above the critical micellar concentration $(\mathrm{cmc}=2.3 \mathrm{~g} / \mathrm{L})$. Unlike solutions in tanks [17], we did not observe any optical activity in thin SDS films, whatever the surfactant concentration or the film thickness. Far above the $\mathrm{cmc}$, the spherical SDS micelles are organized in layers parallel to the film plane (stratification $[18,19]$ ) and are stabilized by structural forces. The effective concentration of the stratified film may be much higher than in solution but its value remains too small to explain such extraordinary optical activity. Glucose glasses were also studied, giving an optical activity of one degree for thicknesses of the order of $1 \mathrm{~mm}$. It is well known that these materials have an amorphous structure [20]. Then the optical activity cannot be due to a sugar amorphous structure in the film.

a)

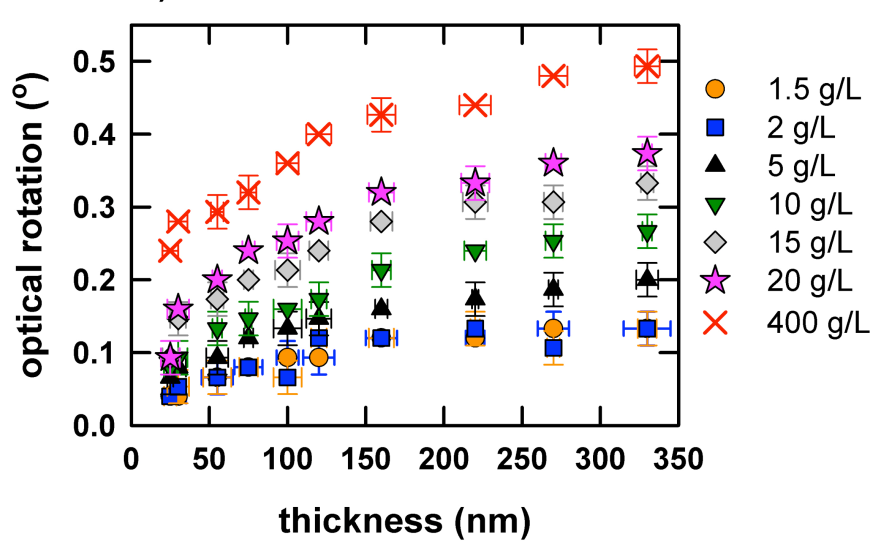

b)

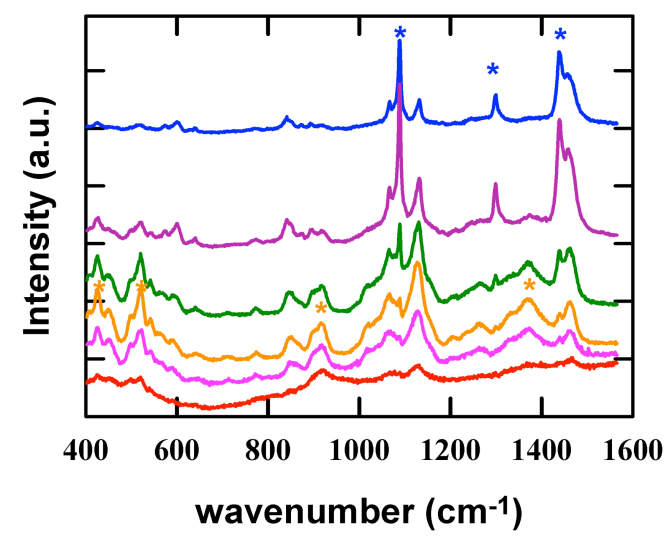

Fig. 1. Optical activity and Raman spectra. (a) Optical rotations of SDS+glucose films at a given $12 \mathrm{~g} / \mathrm{L}$ SDS concentration for various glucose concentrations. (b) Raman spectra of SDS+glucose dried drops for different depths (from 0 to $5 \mu \mathrm{m}$ with a $1 \mu \mathrm{m}$ depth variation). The top spectrum is associated with the upper part of the sample. Blue and orange stars represent SDS and glucose contributions respectively. 
One possible explanation is that this giant optical rotation may be attributed to a particular assembly of chiral molecules within the film and/or at the air/liquid interface. Using synchrotron radiation X-ray scattering, it is now possible to highlight the molecular structure of a thin liquid film [21]. By X-ray diffraction no Bragg peak was observed in the spectra for thin liquid films containing SDS or SDS+glucose mixtures. Their configuration remains amorphous with a lack of a long-range order of SDS micelles. Then, by Raman spectroscopy, we tried to understand the molecular interactions inside the soap films. However, due to the thin thickness probed, the spectra obtained were of no practical use. Nevertheless, to overcome this, studies were made on dried SDS+glucose drops that may have the same conformation at the air/liquid interface (see figure 1.b). SDS is commonly used on the evaporation of aqueous colloidal systems to deposit uniformly particles on the drop surface [22]. We measured the optical activity of these dried drops and surprisingly found that it is also of the order of a few degrees. As the optical activities of SDS+glucose films and dried drops are similar while they are low for large cells containing SDS+glucose solutions, we may assume that interfacial properties for thin liquid films and dried drops are similar. In passing through the SDS+glucose drop from top to bottom, there is a gradual transition from SDS to the phase of glucose in contact with the slide. The spectrum associated with the phase of the SDS is quite similar to that measured on aqueous solutions [23]. Exactly at the middle of the sample, SDS and glucose Raman spectra mix together probably due to the depth of focus $(\sim 2 \mu \mathrm{m})$ of the confocal Raman spectrometer. However, the bands associated with SDS and glucose are not shifted. The spectra of glucose correspond to amorphous states [24]. Neither signature of any assembly nor recombination of SDS via covalent bonds was detected. Nevertheless the optical activity is of the order of a few degrees for SDS+glucose dried drops, whereas the optical activity of glucose dried drops is of the order of 1 degree. This means that there is a peculiar interaction between glucose and SDS both in dried drops and in thin films, probably keeping an amorphous configuration.

\section{Discussion}

Laser light transmission experiments performed with large tanks filled with glucose or SDS+glucose solutions showed no optical activity. SDS and glucose molecules interactions seem to be strongly favored in confined medium. Hydrogen bonding between 
glucose and SDS molecules in self-assembly films has been proposed [25]. Considering the amphiphilic nature of SDS molecules, they must be localized around polar heads $-\mathrm{OSO}_{3}{ }^{-}-$. In order to probe this hypothesis, we used two different surfactant molecules to measure the optical activity of surfactant + glucose in thin films. With TTAB (Tetradecyltrimethylammonium Bromide) that is a cationic surfactant or with Tween 20 that is a neutral surfactant, we hardly observed any optical activity (several orders of magnitude less than for SDS surfactant). With a 50/50 mixture of SDS and TTAB made to ensure neutralization of the head group charges [26], the addition of glucose leads to no optical activity. Such observations confirm that glucose molecules make hydrogen bonds preferably with SDS rather than with water. More generally, the combination of glucose and anionic surfactant having a polar head $-\mathrm{OSO}_{3}{ }^{-}$- would give such a giant optical activity.

Below the cmc (for SDS concentration of $1.8 \mathrm{~g} / \mathrm{L}$ ), we noticed that the optical rotation for the same glucose concentration has the same magnitude. In figure 2.a, the increase of SDS concentration for a fixed glucose concentration $(20 \mathrm{~g} / \mathrm{L})$ leads to no noticeable variation of the optical activity. The optical rotary power is simply controlled by the numbers of films arranged inside the tube (figure 2.a) and is about the same above and below the cmc (figure 2.b). For a fixed SDS+glucose concentration, the more number of films, the bigger the optical activity coupled with the air/liquid interfaces increases. Far above the cmc, there is no significant effect of the contribution of SDS micelles. Moreover, the thickness of the film has little influence on the optical rotation, showing that the giant optical activity mainly originates from the air/liquid interfaces. The addition of dodecanol cosurfactant known to influence the interfacial structure of SDS film [27], dramatically suppresses the optical activity. From these various experimental results, one may thus conclude that (i) glucose molecules interact with polar heads $-\mathrm{OSO}_{3}{ }^{-}$- of SDS molecules and (ii) mainly change conformation at the air/liquid interface of the film and not in the bulk liquid phase. 
a)

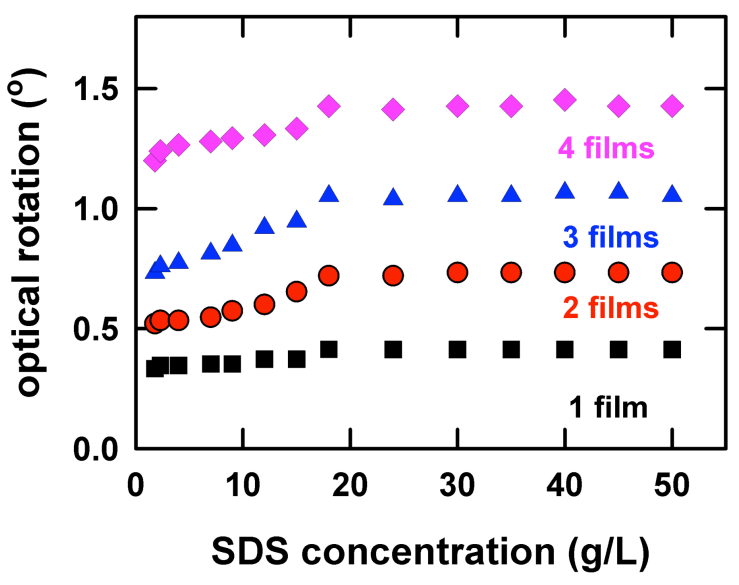

b)

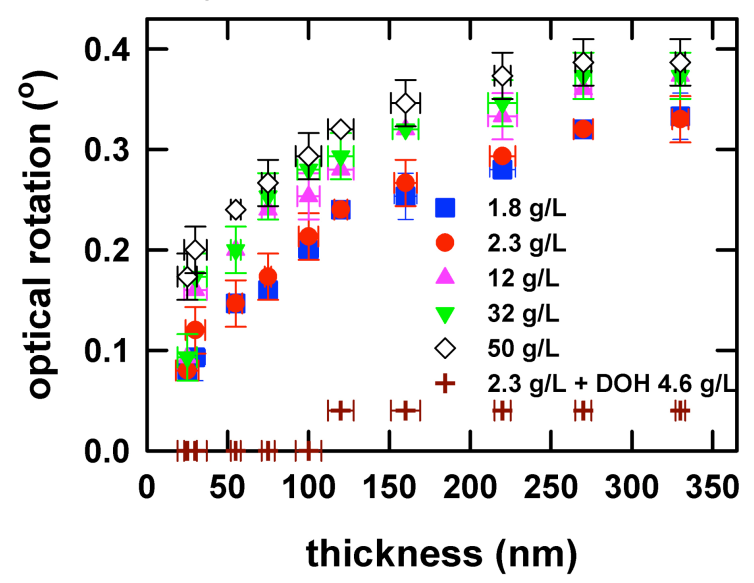

Fig. 2. Optical rotations versus SDS concentration and versus film thickness. (a) Optical rotations for a series of SDS+glucose films with various concentrations, with a $300 \mathrm{~nm}$ thickness, for different numbers of films. The errors in optical rotation are $0.02^{\circ}$. (b) Optical rotations of SDS+glucose films for different SDS concentrations.

\section{Molecular simulations}

To illuminate the molecular mechanisms, we combined experiments with large scale molecular simulations. We carried out molecular dynamic (MD) simulations of bulk liquid phase and air/liquid (AL) interfaces including SDS, $\mathrm{Na}^{+}$, glucose and water molecules. The force field of SDS and $\mathrm{Na}^{+}$was taken from [28]. Water was modelled from the non-rigid and non polarisable TIP4P/2005 model [29]. Glucose was described from AMBER force field [30]. Partial charges of glucose and SDS were extracted from a DFT calculation. A combination of the Becke exchange plus Lee-Yang-Parr correlation functional and allelectron core potentials were used. Additionally, the double-numerical polarization (DNP) basis set was adopted to account for the d-type polarization into heavier atoms and p-type polarization into hydrogen atoms. This basis is similar to the 6-31G(d,p) Gaussian-type basis set. The partial charges were calculated from the Mulliken population analysis. To mimic the experimental conditions we started from a random distribution of molecules in bulk liquid phase. The initial configuration of AL interface consisted of a pre-equilibrated liquid slab surrounded by two empty regions. MD simulations were performed using a time step of $0.002 \mathrm{ps}$ to sample $10 \mathrm{~ns}$ (acquisition phase). The equilibration time corresponds to $10 \mathrm{~ns}$. 
All MD simulations have been carried out with the DL-POLY package [31] using the combination of the Velocity Verlet algorithm and the Nose-Hoover thermostat [32]. Thus, force field and computational procedure of $\mathrm{AL}$ simulations were validated from a comparison in surface tension with respect to the experiment. Mechanical calculation was used to compute surface tension [33].

From MD modelling, we computed free energy profile $\mathrm{F}$ of typical interactions from the pair correlations distributions $(\mathrm{g}(\mathrm{r}))[34]$ as

$\mathrm{F}=-\mathrm{k}_{\mathrm{B}} \mathrm{T} \ln \mathrm{g}(\mathrm{r})$

where $\mathrm{k}_{\mathrm{B}}$ is the Boltzmann's constant and $\mathrm{T}$ the temperature. $\mathrm{F}\left(\right.$ water- $\mathrm{H}_{2} \mathrm{O}$ ), $\mathrm{F}$ (water-SDS) and $\mathrm{F}$ (SDS-glucose) were calculated by considering oxygen atoms of water molecules, anionic group $-\mathrm{OSO}_{3}{ }^{-}$- and glucose molecules. These interacting sites have been chosen because they rule the hydrogen bonds.

The density profiles of SDS, $\mathrm{Na}^{+}$and glucose molecules are reported in figures 3.a. and 3.b. Indeed, as can be seen in figure 3.a, the SDS molecules in bulk phase can be equivalently surrounded by water or by glucose molecules. This could suggest some favourable interactions between interfacial SDS and glucose molecules leading to an interfacial adsorption of glucose. Figure 3.b shows the density profiles of the different molecules between $\mathrm{z}=0 \AA \AA$ and $\mathrm{z}=55 \AA$, $\mathrm{z}$ being the direction that is perpendicular to $\mathrm{AL}$ interfaces. The length of liquid phase is almost $102 \AA$ (between +51 and $-51 \AA$ ) while both AL interfaces are located around $46 \AA$ and $-46 \AA$. Thus, $z=0 \AA$ corresponds to the middle of the film. This figure demonstrates that the glucose molecules are spatially correlated to those of SDS. 
a)

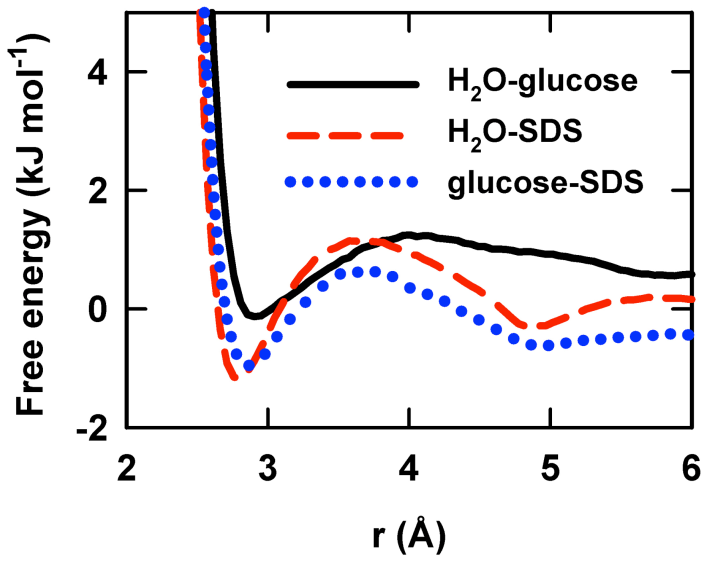

b)

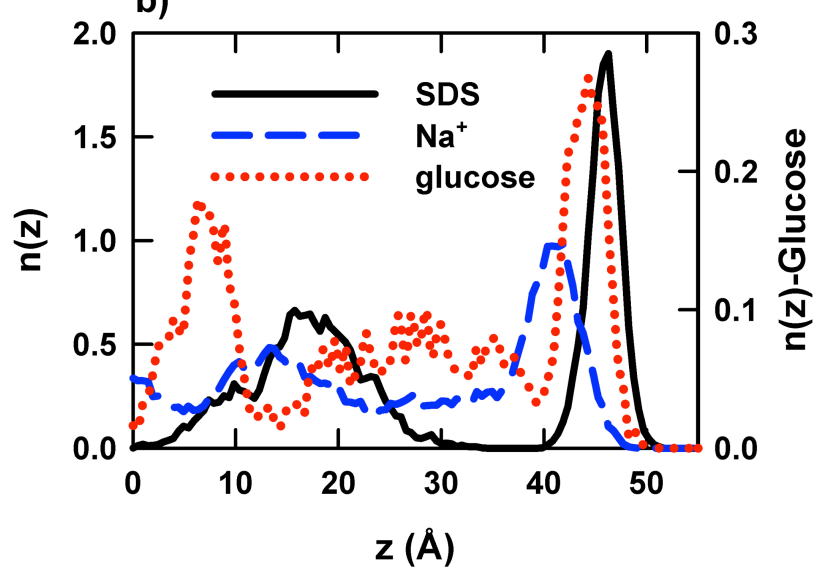

Fig. 3. Calculated free energy profiles and density profiles. (a) Free energy profiles in bulk liquid phase at $298 \mathrm{~K}$ and 1 bar. (b) Density profiles of SDS, $\mathrm{Na}^{+}$(left size) and glucose (right size) in the z-direction that is perpendicular to $\mathrm{AL}$ interfaces.

This bears out our previous conclusions based on free energy profile calculations that suggest some favourable interactions between $-\mathrm{OSO}_{3}{ }^{-}$- and glucose molecules. Thus, some molecules of glucose are anchored close to the interface. This interfacial adsorption suggests a subtle competition between SDS-Na ${ }^{+}$and SDS-glucose interactions. Indeed, in figure 3.b we show that glucose molecules are located in between $\mathrm{Na}^{+}$and SDS molecules and close to them. This peculiar interfacial location could lead to a steric hindrance of glucose inducing a change in its molecular conformation in the presence of SDS and $\mathrm{Na}^{+}$and/or a perturbing influence of the asymmetric environment. From figure $3 b$ it can be noted that i) first of all, the interfacial SDS anchoring is well recovered, ii) $\mathrm{Na}^{+}$is bounded to the anionic groups of SDS from the electrostatic interactions and iii) in liquid phase some SDS molecules selfassemble in micelles (between 10 and $20 \AA$ ). Thus, the underlying physics ruling the microscopic behaviour of surfactants seems to be recovered from MD simulations. This explains the increase in optical activity of sugar through the thin soap film.

\section{Conclusion}

We have demonstrated that the giant optical activity of thin soap films in the presence of sugar is linked to the intramolecular chiral conformation of the sugar molecules at the air/liquid interface. In particular, choosing SDS as typical surfactant in the soap solution and glucose as model sugar molecule, we demonstrated a hydrogen bond interaction 
between the anionic group $-\mathrm{OSO}_{3}{ }^{-}$- and the glucose molecules. Subsequently, a polarization rotator made of a film train seems feasible. It would be also useful to know whether sugar addition could enhance the heat transfer in liquid drops [35] Moreover, it would be interesting to investigate whether the addition of nanoparticles [36,37] could enhance the chiral optical activity. Finally, the optical activity of sugar molecules could be used as a marker to study the dynamics of soap film flows [38].

\section{Acknowledgements}

The authors thank Franck Artzner for valuable help and the ONIS platform (University Rennes 1) for the use of the HR800 Raman spectrometer, and Sean McNamara for careful reading of the manuscript. 


\section{References}

[1] A.L. Kuehner, J. Chem. Educ. 35 (1958) 337.

[2] A.V. Grosse, Science 164 (1969) 291.

[3] C. Isenberg, The science of soap films and soap bubbles, Dover, New York, 1992.

[4] G. Debrégas, P.-G de Gennes, F. Brochard-Wyart, Science 279 (1998) 1704.

[5] H.-L. Jian, X.-X. Liao, L.-W. Zhu, W.-M. Zhang, J.-X. Jiang, J. Colloid Interface Sci. 359 (2011) 487.

[6] J. Emile, M.H.V. Werts, F. Artzner, F. Casanova, O. Emile, J.R.G. Navarro, F. Meneau, J. Colloid Interface Sci. 383 (2012) 124.

[7] G.S. Longo, M. Olvera de la Cruz, I. Szleifer, Soft Matter 8 (2012) 1344.

[8] T. Gibaud, E. Barry, M.J. Zakhary, M. Henglin, A. Ward, Y. Yang, C. Berciu, R. Oldenbourg, M.F. Hagan, D. Nicastro, R.B. Meyer, Z. Dogic, Nature 481 (2012) 348.

[9] J.B. Biot, Mém. Acad. Sci. (France) 13 (1835) 39.

[10] M.D. Ranken, R.C. Kill, C.G.J. Baker, Food Industries Manual, Chapman \& Hall, 1997.

[11] Y.H. Roos, Annu. Rev. Food Sci. Technol 1 (2010) 469.

[12] B.D. Cameron, H.W. Gorde, B. Satheesan, G.L. Cote, Diabetes Technol. Ther. 1 (1999) 135.

[13] F. Chee, T. Fernando, Closed loop control of blood glucose, Springer-Verlag, BerlinHeidelberg, 2007. 
[14] J.T. Suri, D.B. Cordes, F.E. Cappuccio, R.A. Wessling, B. Singaram, Angew. Chem., Int. Ed. 42 (2003) 5857.

[15] T. Kanyanee, J. Jakmunee, K. Grudpan, P.K. Dasgupta, J. Am. Chem. Soc. 132 (2010) 18045.

[16] J. Emile, F. Casanova, G. Loas, O. Emile, Soft Matter 8 (2012) 7223.

[17] A.I. Rusanov, A.G. Nekrasov, Langmuir 26 (2010) 13767.

[18] V. Bergeron, C.J. Radke, Langmuir 8 (1992) 3020.

[19] K.D. Danov, E.S. Basheva, P.A. Kralchevsky, K.P. Ananthapadmanabhan, A. Lips, Adv. Colloid Interf. Sci. 168 (2011) 50.

[20] M.T. Kalichevsky, E.M. Jaroszkiewicz, J.M.V. Blanshard, Int. J. Biol. Macromol. 14, (1992) 257.

[21] J. Emile, S. Pezennec, A. Renault, E. Robert, F. Artzner, C. Meriadec, A. Faisant, F. Meneau, Soft Matter 7 (2011) 9283.

[22] T. Still, P.J. Yunker, A.G. Yodh, Langmuir 28 (2012) 4984.

[23] M. Picquart, J. Phys. Chem. 90 (1986) 243.

[24] S. Söderholm, Y.H. Roos, N. Meinander, M. Hotokka, J. Raman Spectrosc. 30 (1999) 1009.

[25] H. Ji, X. Liu, L. Jin, B. Zhou, X. Yao, X. Wang, Colloids Surf. A 380 (2011) 182.

[26] B. Tah, P. Pal, M. Mahato, G.B. Talapatra, J. Phys. Chem. B 115 (2011) 8493.

[27] E.J. Wanless, T.W. Davey, W.A. Ducker, Langmuir 13 (1997) 4223. 
[28] W.-X. Shi, H.-X. Guo, J. Phys. Chem. B 114 (2010) 6365.

[29] J.L.F. Abascal, C. Vega, J. Chem. Phys. 123 (2005) 234505.

[30] S.J. Weiner, P.A. Kollman, D.A. Case, U.C. Singh, C. Ghio, G. Alagona, S. Profeta, P. Weiner, J. Am. Chem. Soc. 106 (1984) 765.

[31] T.R. Forester, W. Smith, CCP5 Program Library. Daresbury Lab., 2004.

[32] M.P. Allen, D.J. Tildesley, Computer Simulation of Liquids, Clarendon Press, Oxford, 1989.

[33] F. Biscay, A. Ghoufi, V. Lachet, P. Malfreyt, J. Phys. Chem. B 113 (2009) 14277.

[34] N. Rosenbach, H. Jobic, A. Ghoufi, F. Salles, G. Maurin, S. Bourrelly, P.L. Llewellyn, T. Devic, C. Serre, G. Ferey, Ang. Chem. Int. Ed. 47 (2008) 6611.

[35] C. Zhou, J. Han, R. Guo, J. Colloid Interface Sci. 397 (2013) 80.

[36] H.S. Oh, S. Liu, H. Jee, A. Baev, M.T. Swihart, P.N. Prasad, J. Am. Chem. Soc. 132 (2010) 17346.

[37] V. Velasco, S.J. Williams, J. Colloid Interface Sci. 394 (2013) 598.

[38] H. Kellay, Nat. Phys. 7 (2011) 279. 


\section{Glucose vs Thin Soap Film of Glucose}
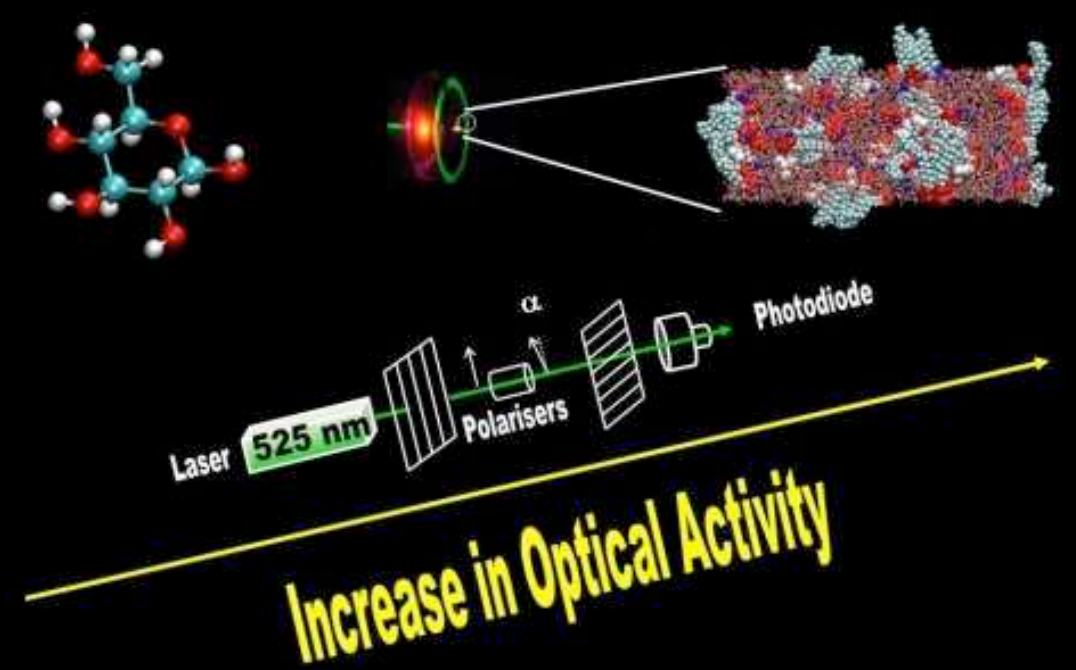


\section{Highlights}

- Optical activity of thin soap films.

- An intramolecular chiral conformation of the sugar molecules at the air/liquid interface.

- Hydrogen bond between the anionic group $-\mathrm{OSO}_{3}{ }^{-}$- of the surfactant SDS and the glucose molecules. 